Ana Paula Machado Lins ${ }^{1}$

Rosely Sichieri ${ }^{2}$

Walmir Ferreira Coutinho ${ }^{3}$

Eloane Gonçalves Ramos ${ }^{4}$

Maria Virginia Marques Peixoto ${ }^{4}$

Vânia Matos Fonseca ${ }^{4}$

${ }^{1}$ Instituto Estadual de Diabetes e Endocrinologia Luiz Capriglione - Grupo de Obesidade e Transtornos Alimentares (IEDE). Endereço: Rua Moncorvo Filho, 90 - Centro, Rio de Janeiro - RJ, 20211-340. anapaulamachadolins@ yahoo.com.br

${ }^{2}$ Instituto de Medicina Social / Departamento de Epidemiologia - UERJ. Endereço: Rua São Francisco Xavier, no 524 CEP.: , Maracanã - RJ. 20550-900

${ }^{3}$ Escola Médica de PósGraduação PUC-RJ no Instituto Estadual de Diabetes e Endocrinologia Luiz Capriglione (IEDE). Endereço: Rua Moncorvo Filho, 90 - Centro, Rio de Janeiro - RJ, 20211-340. ${ }^{4}$ Instituto Fernandes Figueira / Unidade de Pesquisa Clínica (Fiocruz)

\title{
Alimentação saudável, escolaridade e excesso de peso entre mulheres de baixa renda
}

\author{
Healthy eating, schooling and being overweight \\ among low-income women
}

\begin{abstract}
The scope of this study was to analyze the factors associated with the prevalence of being overweight and obesity in a population of lowincome adult women living in a metropolitan region and its association with socioeconomic, demographic, reproductive and lifestyle variables, highlighting the importance of healthy eating. A population-based, cross-sectional study was conducted with a random sample of 758 women aged 20 or older living in Campos Elíseos - Duque de Caxias - State of Rio de Janeiro. Bivariate and multivariate hierarchical regression was used to identify factors associated with overweight and obesity. A prevalence of $23 \%$ of obesity was found, and a prevalence of $56 \%$ of being overweight and obesity combined. An inverse association was found between years of study, being overweight and obesity. Most of the women reported having a healthy diet $(73.6 \%)$ that increased positively with income, education and age. Failure to consume vegetables weekly was associated with being overweight and not having a healthy diet was associated with obesity. The results of this study demonstrate that even in low-income populations, a higher level of education has an impact on prevention of this problem and in food choices.
\end{abstract}

Key words Overweight, Healthy eating, Schooling, Population study
Resumo Objetivo: Analisar os fatores associados à prevalência do excesso de peso e obesidade em uma população de mulheres adultas de baixa renda, moradoras de uma região metropolitana; e sua associação com variáveis socioeconômicas, demográficas, reprodutivas e comportamentais, destacando a alimentação saudável. Métodos: Foi realizado um estudo transversal de base populacional com uma amostra probabilística de $758 \mathrm{mu}$ lheres de 20 anos ou mais moradoras de Campos Elíseos - Duque de Caxias. Utilizou-se análise bivariada e regressão multivariada hierarquizada para identificar fatores associados ao excesso de peso e obesidade. Resultados: Encontrou-se uma prevalência de obesidade de $23 \%$ e prevalências de 56,0\%, quando somados o excesso de peso e a obesidade. Encontrou-se associação inversa entre anos de estudo, excesso de peso e obesidade. A maioria das mulheres referiu alimentação saudável (73,6\%), que aumentou positivamente com a renda, escolaridade e idade. Não consumir semanalmente verduras esteve associado ao excesso de peso e não realizar alimentação saudável esteve associado à obesidade. Conclusões: Os resultados demonstraram que mesmo em uma população com renda baixa, um maior nivel de escolaridade tem impacto na prevenção deste agravo e nas escolhas alimentares.

Palavras-chave Excesso de peso, Alimentação saudável, Escolaridade, Estudo populacional 


\section{Introdução}

O Brasil tem realizado inquéritos em intervalos que permitem conhecer e comparar a evolução dos agravos nutricionais mais relevantes, bem como a distribuição regional dos mesmos. A análise dos resultados desses estudos aponta a ascensão da obesidade, interpretada como um reflexo das transformações ocorridas nos âmbitos da economia, condições de vida, saúde e nutrição da população brasileira nas últimas décadas ${ }^{1}$.

De acordo com dados recentes da Pesquisa de Orçamentos Familiares (POF - 2008-2009), 64,9\% das mulheres brasileiras apresentam algum grau de excesso de peso, quando somados o excesso de peso e a obesidade, o que demonstra a magnitude deste agravo na população feminina ${ }^{2}$.

De uma maneira geral, os grandes inquéritos nacionais e os estudos regionais têm demonstrado diferenciação de gênero no agravo em questão, além de variações de faixa etária, área geográfica e renda ${ }^{1,2}$, configurando a obesidade como um fenômeno predominantemente urbano com tendência de aumento nas áreas rurais, mais expressivo nas regiões sul e sudeste do país e bastante significativo entre mulheres inseridas nos estratos de menor renda ${ }^{2-4}$.

Múltiplos fatores estão envolvidos no desenvolvimento da obesidade, sendo os relativos ao meio ambiente, os socioeconômicos e os demográficos considerados principais ${ }^{5}$.

Na contramão de um estilo de vida saudável, a obesidade tem sido associada a um maior consumo de alimentos com alta densidade calórica, ricos em gordura saturada e açúcar, somada à diminuição do consumo de carboidratos complexos, fibras e da realização de atividade física no lazer ${ }^{2,6}$.

No Brasil, a exemplo do que acontece em outros países do mundo, o padrão alimentar atual inclui ainda um consumo insuficiente de frutas, legumes e verduras (FLV), padrão este associado não somente à obesidade como a outras patologias ${ }^{7-9}$.

O consumo insuficiente de FLV está entre os principais fatores de risco para a carga global de doença em todo o mundo, uma vez que são considerados importantes na composição de uma alimentação saudável, agindo de maneira inquestionável na manutenção da saúde e no balanço energético, introduzindo nutrientes com efeitos significativos, especificamente prevenindo diversos tipos de câncer e Doenças Crônicas não Transmissíveis (DCNT) como obesidade, diabetes tipo 2 e doenças cardiovasculares s,10-12. $^{8}$.
Atualmente diversas iniciativas no campo das políticas públicas voltadas para a melhoria da alimentação e nutrição das populações em vários países do mundo propõem um conjunto de intervenções integradas que enfrentam o desafio de promover mudanças nos hábitos alimentares dos indivíduos e suas famílias, levando em consideração, principalmente, as práticas alimentares regionais, alimentos minimamente processados, culturalmente referenciados, acessíveis e de elevado valor nutritivo ${ }^{13}$.

A Estratégia Global sobre Alimentação Saudável, Atividade Física e Saúde ${ }^{13}$ e o Guia Alimentar para a População Brasileira ${ }^{14}$, são exemplos de diretrizes alimentares oficiais voltadas para promoção da alimentação saudável visando à redução da incidência de doenças crônicas.

Apesar de uma grande quantidade de estudos voltados para a investigação dos padrões de consumo alimentar dos diversos grupos populacionais, são escassos aqueles que procuram conhecer a opinião das pessoas sobre sua alimentação.

O presente estudo tem como objetivo descrever os fatores associados à prevalência de excesso de peso e obesidade em uma população de mulheres adultas e pobres, moradoras de Campos Elíseos, em Duque de Caxias (RJ); e sua associação com variáveis socioeconômicas, demográficas, reprodutivas e comportamentais, destacando a alimentação saudável referida pelas mulheres como a variável principal neste contexto.

\section{Material e Métodos}

Trata-se de estudo transversal de base populacional que faz parte de um projeto maior intitulado "Avaliação do estado nutricional, hábitos alimentares e insegurança alimentar no município de Duque de Caxias, Rio de Janeiro: desenvolvimento de um instrumento simplificado para avaliação de consumo alimentar saudável”, realizado em Campos Elíseos com financiamento do Conselho Nacional de Desenvolvimento Científico e Tecnológico (CNPQ) e do Instituto Nacional do Câncer (INCA).

\section{Plano de amostragem}

Para realização do estudo original, foi utilizada uma amostra probabilística de 1.125 domicílios particulares permanentes (DPP) de Campos Elíseos, selecionada em três estágios (setor censitário, domicílio e o indivíduo). O tamanho da 
amostra foi fixado com base em uma estimativa de $14,5 \%$ para a prevalência de pobreza extrema, fixando-se uma margem de erro de 5,0\%. A prevalência atual de sobrepeso e obesidade somados é em torno de $50 \%$ entre as mulheres brasileiras.

O tamanho final da amostra foi de 1.085 domicílios, com 3,4\% sem resposta. Para o presente estudo, foram selecionadas as mulheres de 20 anos ou mais não grávidas e nem amamentando no momento da entrevista totalizando 758 mulheres.

\section{Variáveis analisadas}

As medidas antropométricas obtidas segundo as recomendações ${ }^{15}$ foram categorizadas em normal $($ IMC $>18)$, sobrepeso $($ IMC $>25)$ e obesidade (IMC $>30$ ).

Renda per capita do domicílio. Total de rendimentos da família dividido pelo número de moradores, expressa em relação ao salário mínimo (SM) e categorizada até $1 / 4$ do SM, de $1 / 4$ a meio $\mathrm{SM}$, de meio a $1 \mathrm{SM}$ e acima de $1 \mathrm{SM}$.

Atividade Física Laboral. Atividade exercida pela entrevistada em sua ocupação no momento da entrevista, classificada conforme o documento oficial ${ }^{16}$ e a Portaria $n^{\circ}$. 3.214/78 (Ministério do Trabalho $)^{17}$ em: atividade leve; atividade moderada/pesada e sem ocupação.

Atividade física de lazer e doméstica. Foi avaliada através de perguntas referentes à realização nos últimos três meses das atividades físicas realizadas no lazer (caminhada, vôlei, musculação, ginástica, hidroginástica, bicicleta, corrida, futebol, lutas, natação, basquete, outra atividade referida) e diárias (deslocamento para o trabalho, cuidados com criança menor de três anos e outros trabalhos domésticos), com informação da duração em minutos e frequência semanal. O tempo gasto em cada atividade foi multiplicado por uma taxa metabólica equivalente (MET) específica para cada atividade gerando as classificações: inativo, levemente ativo e ativo ${ }^{18}$.

Tabagismo. Foi considerado fumante quem consumiu mais de 100 cigarros durante a vida e respondeu fumar ainda no momento da entrevista; ex-fumante, a pessoa que afirmou ter fumado mais de 100 cigarros e não fumava mais no momento da entrevista e não-fumante quem respondeu nunca ter fumado, ou que fumou menos de 100 unidades durante a vida.

Etilismo. Frequência semanal categorizada em menos de $1 \mathrm{vez} / \mathrm{semana}, 1 \mathrm{vez} / \mathrm{semana}$, maior ou igual a 2 vezes/semana e não consome.

Segurança Alimentar. Utilizou-se questionário de avaliação de segurança e insegurança ali- mentar familiar brasileira - Escala Brasileira de Insegurança Alimentar (EBIA) ${ }^{19}$, criadas as categorias: insegurança e segurança alimentar.

Consumo Semanal de Verduras e Legumes. Categorizada como sim ou não, obtida a partir da pergunta sobre consumo todos os dias ou quase todos os dias - pelo menos 5 dias da semana (consumo regular).

Consumo Semanal de Frutas. Categorizada como sim ou não, obtida a partir da pergunta sobre consumo de frutas, todos os dias ou quase todos os dias - pelo menos 5 dias da semana (consumo regular).

Alimentação Saudável Autorreferida. Categorizada como sim ou não, obtida a partir da pergunta sobre a opinião da entrevistada a respeito da sua alimentação, saudável ou não.

\section{Análise de dados}

Todos os procedimentos de análise utilizaram os pesos amostrais calibrados e informações do plano amostral. Inicialmente, foram realizadas análises bivariadas entre a variável resposta e cada variável explicativa, utilizando como medida de efeito a razão de prevalência. Somente as variáveis explicativas que apresentaram $p$ valor $<0,2$ ou que possuíssem evidência de associação com a variável resposta relatada na literatura foram incluídas na análise multivariada.

Para a análise multivariada, utilizou-se uma abordagem hierárquica ${ }^{20}$. Os níveis de hierarquia foram distribuídos em bloco distal (variáveis socioeconômicas e demográficas), bloco intermediário (reprodutivas) e proximal (comportamentais - hábitos alimentares) (Figura 1). O modelo foi ajustado para aquelas variáveis que se encontravam no mesmo nível ou em nível imediatamente superior.

Em cada bloco hierárquico, as variáveis foram incluídas no modelo, uma de cada vez, em ordem decrescente do grau de associação com a variável resposta, conforme obtido nas análises bivariadas. Para cada variável incluída, verificava-se se os valores de $p$ dos coeficientes estimados eram menores que 0,05 .

Utilizou-se o modelo Log-Log Complementar, considerado uma alternativa mais adequada ao Modelo de Regressão Logística para estimar a Razão de Prevalência em estudos transversais com desfecho frequentes e altas prevalências ${ }^{21,22}$.

O projeto de pesquisa foi aprovado pelo Comitê de Ética e Pesquisa da Universidade do Estado do Rio de Janeiro. No momento da entrevista domiciliar, foi apresentado o Termo de Consen- 
timento livre e esclarecido, no qual todos os participantes assinaram após esclarecimentos quanto aos procedimentos a serem empregados, a garantia de sigilo das informações prestadas e a possibilidade de se recusar a participar da investigação antes de sua inclusão na amostra.

\section{Resultados}

A população total de mulheres com 20 anos ou mais, residentes no município de Campos Elíseos - Duque de Caxias, foi estimada a partir da amostra em 56.880 (Coeficiente de Variação $\mathrm{CV}=4,7 \%$ ). A distribuição de cada variável analisada se encontra na Tabela 1 . Do total das mulheres, apenas 3,6\% apresentaram baixo peso, $40,0 \%$ foram classificadas como normais, 33,0 \% apresentaram excesso de peso e $23,0 \%$ obesidade, ou seja, mais da metade das mulheres (56,3\%) estava acima do peso. Boa parte $(37,0 \%)$ possuía até quatro anos de estudo. Apenas 5,0 \% relataram renda per capita acima de dois salários mínimos e 19,0 \% até um salário mínimo. A

\section{Bloco distal}

$$
\begin{gathered}
\text { Socioeconômicas } \\
\text { Anos de estudo } \\
\text { Renda per capita }
\end{gathered}
$$

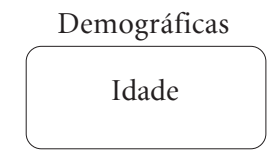

Bloco intermediário

\begin{tabular}{|c|c|}
\hline Comportamentais & Hábitos alimentares \\
\hline $\begin{array}{c}\text { Atividade física laboral } \\
\text { Atividade física de lazer } \\
\text { e doméstica } \\
\text { Consumo de álcool }\end{array}$ & $\begin{array}{c}\text { Segurança alimentar } \\
\text { Consumo semanal de } \\
\text { verduras e legumes } \\
\text { Consumo semanal de } \\
\text { frutas } \\
\text { Alimentação saudável } \\
\text { auto-referida }\end{array}$ \\
\hline $\begin{array}{l}\text { Sobr } \\
\text { Obes }\end{array}$ & \\
\hline
\end{tabular}

Reprodutivas
Menopausa
Número de filhos
Idade da menarca

\section{Bloco proximal}

Figura 1. Modelo hierárquico conceitual. maior parte de mulheres que realizou atividades laborais moderadas referiu exercer atividade como empregada doméstica (32,0\%) e faxineira $(22,0 \%)$ e $69,5 \%$ referiram não ter ocupação no momento da entrevista. Dentre as mulheres que exerciam atividade laboral leve, $62,0 \%$ possuíam mais do que 8 anos de estudo. Para aquelas que exerciam atividade laboral moderada este percentual cai para 33,0 \%.

Os dados relativos à atividade física de lazer demonstraram que a grande maioria $(78,8 \%)$ das mulheres era inativa; porém quando somadas as atividades exercidas no lazer e as atividades exercidas no dia a dia, apenas $3,7 \%$ foram classificadas como inativas e $86,2 \%$ classificadas como ativas.

Uma boa parte declarou-se não fumante $(69,6 \%)$ e não consumidora de bebida alcoólica $(68,4 \%)$. Aproximadamente a metade da amostra $(56,2 \%)$ referiu algum grau de insegurança alimentar.

A maior parte das mulheres referiu ter uma alimentação saudável $(73,6 \%)$, hábito que aumentou positivamente em relação à renda. Quanto ao consumo de verduras, $80,2 \%$ referiram sim, enquanto em relação ao consumo de frutas apenas a metade do grupo respondeu positivamente $(54,1 \%)$.

A partir da análise bivariada, observa-se um aumento da obesidade a partir dos 40 anos de idade em relação às faixas anteriores $(26 \%, \mathrm{RP}$ 2,61 - IC 95\%: 1,30 - 5,30) (Tabela 1). Este aumento, porém, foi ainda mais expressivo para aquelas a partir dos 50 anos (37,8\%, RP: 3,80 IC 95\%: 1,92 - 7,51) (Tabela 1), um aumento confirmado na análise multivariada (RP: 3,32 IC 95\%: 1,52 - 7,23).

Em relação à escolaridade, as prevalências de obesidade foram maiores entre aquelas que não estudaram ou estudaram poucos anos (RP: 2,44 - IC 95\%: 1,57 - 3,80) (Tabela 1). Destaca-se que não houve diferença significativa nas prevalências de excesso de peso em relação aos anos de estudo; porém, os resultados da análise multivariada demonstraram que para aquelas com 0-4 anos de estudo a razão de prevalência para o sobrepeso foi 1,70 vezes maior em relação àquelas que estudaram acima de oito anos (RP: 1,70 - IC 95\%: 1,14 - 2,52) (Tabela 2), enquanto que a obesidade, na comparação destas mesmas faixas de escolaridade, foi de 1,95 (RP: 1,95 - IC 95\%: 1,08 - 3,52) (Tabela 2).

Quanto às características reprodutivas, mulheres na menopausa apresentaram 1,58 vezes mais probabilidade de apresentar obesidade em 
Tabela 1. Prevalência (\%) de categorias do estado nutricional segundo características demográficas, socioeconômicas, reprodutivas, comportamentais e razão de prevalência (RP) de sobrepeso e obesidade em mulheres. Duque de Caxias - RJ, 2005.

\begin{tabular}{|c|c|c|c|c|c|c|}
\hline \multirow[b]{2}{*}{ Variáveis } & \multirow[b]{2}{*}{$\mathbf{n}$} & \multicolumn{2}{|c|}{ Excesso de peso } & \multicolumn{2}{|r|}{ Obesidade } & \multirow[b]{2}{*}{ p valor } \\
\hline & & $(\%)$ & RP (IC95\%) & $(\%)$ & RP (IC95\%) & \\
\hline \multicolumn{7}{|l|}{ Socioeconômicas e demográficas } \\
\hline Faixa etária & & & & & & $<0,01$ \\
\hline $20-29$ & 13.441 & 35,9 & 1 & 9,9 & 1 & \\
\hline $30-39$ & 14.126 & 32,7 & $0,91(0,59-1,41)$ & 15,9 & $1,60 \quad(0,74-3,43)$ & \\
\hline $40-49$ & 9.259 & 34,0 & $0,94(0,60-1,51)$ & 26,0 & $2,61 \quad(1,30-5,30)$ & \\
\hline $50-59$ & 11.192 & 29,6 & $0,82(0,50-1,36)$ & 37,8 & $3,80 \quad(1,92-7,51)$ & \\
\hline Anos de Estudo & & & & & & $<0,01$ \\
\hline $0-4$ & 20.328 & 33,9 & $0,98(0,65-1,50)$ & 30,0 & $2,44(1,57-3,80)$ & \\
\hline $5-7$ & 13.326 & 30,9 & $0,90(0,59-1,40)$ & 28,0 & $2,28(1,36-3,81)$ & \\
\hline 8 ou mais & 21.389 & 34,3 & 1 & 12,3 & 1 & \\
\hline $\begin{array}{l}\text { Renda per capita em salários } \\
\text { mínimos * }\end{array}$ & & & & & & $<0,01$ \\
\hline Até $1 / 4$ & 9.871 & 49,0 & $1,27(0,80-2,02)$ & 16,7 & $0,63(0,32-1,21)$ & \\
\hline $1 / 4$ a $1 / 2$ & 17.153 & 20,9 & $0,54(0,33-0,87)$ & 21,2 & $0,80(0,45-1,40)$ & \\
\hline $1 / 2$ a 1 & 17.637 & 34,6 & $0,90(0,60-1,36)$ & 23,6 & $0,88(0,52-1,50)$ & \\
\hline Acima de 1 & 10.256 & 38,5 & 1 & 26,6 & 1 & \\
\hline \multicolumn{7}{|l|}{ Reprodutivas } \\
\hline Menopausa & & & & & & 0,01 \\
\hline $\operatorname{Sim}$ & 20.238 & 36,0 & $1,11(0,82-1,50)$ & 30,2 & $1,58(1,10-2,25)$ & \\
\hline Não & 34.354 & 32,4 & 1 & 19,2 & 1 & \\
\hline Número de Filhos & & & & & & 0,06 \\
\hline 0 & 405 & 98,9 & $3,72(2,50-5,60)$ & 1,1 & $0,05(0,00-0,78)$ & \\
\hline 1 & 11.522 & 26,5 & 1 & 19,7 & 1 & \\
\hline 2 & 14.975 & 34,0 & $1,29(0,80-2,03)$ & 19,9 & $1,00(0,58-1,76)$ & \\
\hline 3 & 10.536 & 38,7 & $1,45(0,86-2,45)$ & 23,0 & $1,16(0,62-2,17)$ & \\
\hline 4 ou mais & 12.391 & 29,8 & $1,12(0,64-1,94)$ & 35,4 & $1,80(1,04-3,10)$ & \\
\hline Idade da Menarca (anos) & & & & & & 0,04 \\
\hline $8-11$ & 8.095 & 31,7 & $1,13(0,73-1,73)$ & 35,1 & $1,50(0,96-2,34)$ & \\
\hline $11-13$ & 27.367 & 37,6 & $1,33(0,99-1,80)$ & 19,2 & $0,82(0,52-1,30)$ & \\
\hline 14 ou mais & 18.517 & 28,1 & 1 & 23,4 & 1 & \\
\hline
\end{tabular}

relação àquelas que ainda não haviam entrado na menopausa e menstruavam (Tabela 1); contudo, na análise multivariada este efeito desapareceu (Tabela 2).

A renda per capita entre $1 / 4$ e $1 / 2$ salário mínimo teve efeito protetor para o excesso de peso e as outras faixas de renda não estiveram associadas nem a este desfecho e nem à obesidade.

Mulheres que não tiveram filhos apresentaram praticamente 4 vezes menos chance de excesso de peso do que aquelas que tiveram pelo menos 1 filho (IC 95\%: 2,50 - 5,60), resultado que permanece na análise multivariada (RP: 4,94 - IC 95\%: 1,81 - 13,4) (Tabela 2). Enquanto não ter filhos pareceu ser um fator de proteção para a obesidade, ter quatro ou mais filhos represen- tou uma chance quase duas vezes maior para este desfecho (Tabela 1).

De acordo com a análise bivariada, as características da atividade física de lazer e doméstica, assim como o etilismo e o tabagismo, não estiveram associadas ao desfecho. Apenas a atividade física laboral leve teve efeito protetor para o excesso de peso (Tabela1).

Por outro lado, de acordo com os resultados da análise multivariada, exercer atividade física de lazer e doméstica, pelo menos de maneira leve, parece ter efeito protetor para o excesso de peso (RP: 0,38 - IC 95\%: 0,17 - 0,83) (Tabela 2).

Em torno de $74,0 \%$ das mulheres referiram realizar uma alimentação saudável, consumindo semanalmente mais verduras $(80,0 \%)$ do que fru- 


\begin{tabular}{|c|c|c|c|c|c|c|c|}
\hline \multicolumn{8}{|l|}{362} \\
\hline \multirow{2}{*}{\multicolumn{8}{|c|}{ Tabela 1 . continuação }} \\
\hline & & & & & & & \\
\hline & \multirow[b]{2}{*}{ Variáveis } & \multirow[b]{2}{*}{$\mathbf{n}$} & \multicolumn{2}{|r|}{ Excesso de peso } & \multicolumn{2}{|r|}{ Obesidade } & \multirow[b]{2}{*}{$\mathrm{p}$ valor } \\
\hline & & & $(\%)$ & RP (IC95\%) & $(\%)$ & RP (IC95\%) & \\
\hline & \multicolumn{7}{|l|}{ Comportamentais } \\
\hline & Atividade Física Laboral & & & & & & 0,06 \\
\hline & Moderada/Pesada & 8.496 & 38,9 & 1 & 27,4 & 1 & \\
\hline & Leve & 8.523 & 22,2 & $0,57(0,33-0,97)$ & 19,3 & $0,70(0,33-1,50)$ & \\
\hline & Sem Ocup. & 38.741 & 34,4 & $0,88(0,64-1,22)$ & 22,8 & $0,83(0,57-1,21)$ & \\
\hline & $\begin{array}{l}\text { Atividade Física de Lazer e } \\
\text { Doméstica }\end{array}$ & & & & & & 0,97 \\
\hline & Ativo & 47.627 & 33,5 & 1 & 22,2 & 1 & \\
\hline & Lev. Ativo & 5.467 & 33,5 & $0,60(0,33-1,01)$ & 27,0 & $1,07(0,50-2,30)$ & \\
\hline & Inativo & 2.090 & 33,3 & $0,44(0,17-1,13)$ & 22,3 & $1,50(0,81-2,71)$ & \\
\hline & Etilismo & & & & & & 0,43 \\
\hline & Não & 37.869 & 33,4 & 1 & 23,9 & 1 & \\
\hline & $<1 \mathrm{vez} / \mathrm{sem}$ & 7.216 & 27,0 & $0,80(0,56-1,15)$ & 18,8 & $0,78(0,46-1,34)$ & \\
\hline & $1 \mathrm{vez} / \mathrm{sem}$ & 6.225 & 43,9 & $1,31(0,87-1,97)$ & 24,2 & $1,01(0,54-1,90)$ & \\
\hline & $>2$ vezes/sem & 3.997 & 29,2 & $0,87(0,43-1,75)$ & 22,2 & $0,92(0,51-1,66)$ & \\
\hline & Tabagismo & & & & & & 0,57 \\
\hline & Fumante & 8.639 & 34,7 & 1 & 18,8 & 1 & \\
\hline & Ex-fumante & 7.724 & 30,1 & $0,86(0,50-1,51)$ & 31,8 & $1,70(0,83-3,45)$ & \\
\hline & Não fumante & 38.374 & 33,5 & $0,96(0,67-1,38)$ & 21,8 & $1,16(0,62-2,14)$ & \\
\hline & \multicolumn{7}{|l|}{ Hábitos Alimentares } \\
\hline & Segurança Alimentar & & & & & & 0,97 \\
\hline & Insegurança & 31.494 & 33,7 & 1 & 22,7 & 1 & \\
\hline & Segurança & 24.250 & 32,6 & $1,03(0,74-1,44)$ & 23,4 & $0,96(0,66-1,42)$ & \\
\hline & \multicolumn{6}{|l|}{ Verduras } & 0,13 \\
\hline & Sim & 43.983 & 30,9 & 1 & 23,6 & 1 & \\
\hline & Não & 10.982 & 41,5 & $1,34(1,01-1,80)$ & 19,2 & $0,81(0,53-1,24)$ & \\
\hline & Consumo Semanal de Frutas & & & & & & 0,92 \\
\hline & Sim & 29.583 & 34,1 & 1 & 22,0 & 1 & \\
\hline & Não & 25.577 & 32,4 & $0,95(0,67-1,33)$ & 23,4 & $1,06(0,72-1,60)$ & \\
\hline & $\begin{array}{l}\text { Alimentação Saudável } \\
\text { autorreferida }\end{array}$ & & & & & & 0,04 \\
\hline & Sim & 40.429 & 33,6 & 1 & 19,1 & 1 & \\
\hline & Não & 14.507 & 32,1 & $0,95(0,66-1,36)$ & 31,8 & $1,66(1,16-2,37)$ & \\
\hline
\end{tabular}

* O valor do salário mínimo (SM) no ano de 2005, no Estado do Rio de Janeiro, foi igual a R\$373,00.

tas $(54,0 \%)$. Destas, $64,0 \%$ que referiram não realizar uma alimentação saudável estavam acima do peso, quando somadas as prevalências do excesso de peso e obesidade.

A alimentação saudável e o consumo de frutas e verduras aumentaram sua prevalência a partir dos 50 anos $(83,0 \%, 64,1 \%$ e $85,2 \%$, respectivamente), acima dos 8 anos de estudo $(79,2 \%, 61,7 \%$ e $83,4 \%$, respectivamente) e acima de 1 salário mínimo $(88,4 \%, 72,9 \%$ e $89,0 \%$, respectivamente).

Não consumir semanalmente verduras esteve associado ao sobrepeso (RP: 1,34 - IC 95\%:
$1,01-1,80$ ), assim como não realizar alimentação saudável esteve associada à obesidade (RP: 1,66 - IC 95\%: 1,16 - 2,37) (Tabela 1).

Dentre as mulheres que referiram alimentação saudável, a maior parte afirmou realizar em média três refeições por dia $(79,2 \%)$, comer arroz e feijão todos os dias da semana $(73,8 \%$ e $74,5 \%$, respectivamente) e consumir regularmente verduras e frutas $(79,6 \%$ e $89,8 \%$ respectivamente) (Tabela 3).

A associação da alimentação saudável tanto para o excesso de peso quanto para a obesidade permaneceu significante no modelo (Tabela 2). 
Tabela 2. Modelo hierarquizado final, com as razões de prevalência (RP) e os intervalos de confiança (IC) de 95\% associadas ao sobrepeso e obesidade em mulheres. Duque de Caxias - RJ, 2005.

\begin{tabular}{|c|c|c|c|c|}
\hline \multirow{2}{*}{ Variáveis selecionadas } & \multicolumn{2}{|c|}{ Excesso de peso } & \multicolumn{2}{|c|}{ Obesidade } \\
\hline & RP & IC 95\% & $\mathbf{R P}$ & IC 95\% \\
\hline \multicolumn{5}{|l|}{ Bloco I e demográficas } \\
\hline \multicolumn{5}{|l|}{ Faixa etária } \\
\hline $20-29$ & 1 & - & 1 & - \\
\hline $30-39$ & 1,34 & $0,83-2,17$ & 1,55 & $0,78-3,09$ \\
\hline $40-49$ & 1,56 & $0,92-2,63$ & 1,93 & $0,98-3,78$ \\
\hline $50-59$ & 2,34 & $1,34-4,10$ & 3,32 & $1,52-7,23$ \\
\hline \multicolumn{5}{|l|}{ Anos de Estudo } \\
\hline 8 ou mais & 1 & - & 1 & - \\
\hline $5-7$ & 1,36 & $0,89-2,07$ & 1,68 & $0,85-3,29$ \\
\hline $0-4$ & 1,70 & $1,14-2,52$ & 1,95 & $1,08-3,52$ \\
\hline \multicolumn{5}{|l|}{ Bloco II } \\
\hline \multicolumn{5}{|l|}{ Idade da Menarca } \\
\hline 8-11 anos & 1,93 & $1,18-3,13$ & 2,45 & $1,22-4,90$ \\
\hline $11-13$ anos & 1,22 & $0,84-1,77$ & 1,06 & $2,05-0,55$ \\
\hline 14 anos ou mais & 1 & - & 1 & - \\
\hline \multicolumn{5}{|l|}{ Número de Filhos } \\
\hline nenhum filho & 4,94 & $1,81-13,4$ & 4,48 & $0,87-23,0$ \\
\hline 1 filho & 1 & - & 1 & - \\
\hline 2 filhos & 0,91 & $0,53-1,57$ & 0,83 & $0,40-1,72$ \\
\hline 3 filhos & 1,01 & $0,55-1,84$ & 0,92 & $0,41-2,03$ \\
\hline 4 ou mais filhos & 0,85 & $0,49-1,49$ & 0,74 & $0,31-1,79$ \\
\hline \multicolumn{5}{|l|}{ Bloco III } \\
\hline \multicolumn{5}{|c|}{ Atividade física de lazer e doméstica } \\
\hline ativo & 1 & - & 1 & - \\
\hline levemente ativo & 0,38 & $0,17-0,83$ & 0,38 & $0,08-1,71$ \\
\hline inativo & 0,99 & $0,38-2,59$ & 2,43 & $0,60-9,83$ \\
\hline \multicolumn{5}{|c|}{ Alimentação saudável autorreferida } \\
\hline Sim & 1 & - & 1 & - \\
\hline Não & 1,56 & $1,11-2,20$ & 1,60 & $1,05-2,46$ \\
\hline
\end{tabular}

Bloco I - ajustado para Faixa etária e Anos de estudo; Bloco II - ajustado para Faixa etária, Anos de estudo, Idade da menarca e Número de filhos; Bloco III - ajustado para ajustado para Faixa etária, Anos de estudo, Idade da menarca, Número de filhos,

Atividade física de lazer e doméstica e Alimentação saudável.

\section{Discussão}

A prevalência de obesidade $(23 \%)$ encontrada entre as mulheres de Campos Elíseos foi maior em relação aos resultados encontrados recentemente para as regiões Sul $(19,3 \%)$ e Sudeste $(17,4 \%)^{2}$

No entanto, quando somados o excesso de peso e obesidade, apesar de $56 \%$ das mulheres estudadas estarem acima do peso, este valor esteve abaixo da média nacional $(64,9 \%)^{2}$, mas consistente com a Pesquisa Nacional de Demografia e Saúde, que encontrou prevalências de 59,0\% entre mulheres adultas brasileiras ${ }^{23}$. Outros estudos com resultados semelhantes ${ }^{24-26}$ demonstra- 
vezes menor em relação às mulheres da região sudeste $(58,0 \%)^{30}$.

Como a faixa de variação de renda na população estudada não é grande, não foi encontrada associação entre renda e obesidade. Contudo, outros estudos têm demonstrado que a escolaridade mais do que a renda é o fator associado ${ }^{31}$.

Igualmente consistente com a literatura foi a associação inversa encontrada entre anos de estudo, sobrepeso e obesidade. Ao analisar dados de inquéritos nacionais, observa-se o nível de escolaridade como variável principal responsável pela associação inversa entre nível socioeconômico e obesidade entre mulheres brasileiras ${ }^{32}$

Segundo Teichmann et al. ${ }^{24}$, esta associação pode ser atribuída, em parte, aos efeitos de pouca informação e orientação para a perda de peso, configurando um estilo de vida menos saudável, onde há aumento do consumo de alimentos de baixo custo e alta densidade energética. Embora o grupo estudado apresente níveis de escolaridade mais baixos e exerça atividades como: domésticas $(32,0 \%)$ e faxineiras $(22,0 \%)$, os resultados mostraram que mesmo a pouca escolaridade do referido grupo foi fator de proteção para o sobrepeso e obesidade, principalmente quando somada a característica global de uma dieta saudável.

A maior parte das mulheres respondeu positivamente quanto ao consumo semanal de verduras (80,0\%) e de alimentação saudável (74,0\%), enquanto o consumo de frutas foi relatado por metade do grupo estudado. Aquelas que referiram alimentação saudável realizaram três refeições por dia $(79,2 \%)$, consumiram arroz e feijão todos os dias da semana $(73,8 \%$ e $74,5 \%$, respectivamente) e consumiram regularmente verduras e frutas $(79,6 \%$ e $89,8 \%$ respectivamente) (Tabela 3), quantidade e qualidade compatível com uma alimentação saudável ${ }^{14}$, demonstrando que a percepção destas mulheres em relação à qualidade da sua alimentação corresponde ao esperado.

O consumo encontrado entre as mulheres de Campos Elíseos foi maior em relação aos achados de diversos estudos ${ }^{33-36}$, embora a forma de avaliar tenha sido muito diferente. A resposta positiva em relação à alimentação saudável mostrou concordância com o sobrepeso e a obesidade destas mulheres. De acordo com a análise multivariada, para aquelas que referiram não realizar uma alimentação saudável a chance de sobrepeso e obesidade aumentou 1,56 e 1,60 vezes, respectivamente.

Ao analisar os dados de 2006 do Sistema de Vigilância de Fatores de Risco e Proteção para
Tabela 3. Frequência de consumo de alimentos correlatos à alimentação saudável entre mulheres. Duque de Caxias - RJ, 2005.

\begin{tabular}{|c|c|c|}
\hline \multirow[t]{2}{*}{ Variáveis } & \multicolumn{2}{|c|}{$\begin{array}{c}\text { Alimentação } \\
\text { saudável } \\
\text { autorreferida }\end{array}$} \\
\hline & $\begin{array}{l}\text { Sim } \\
(\%)\end{array}$ & $\begin{array}{c}\text { Não } \\
(\%)\end{array}$ \\
\hline \multicolumn{3}{|l|}{ Número de refeições por dia } \\
\hline 1 & 42,2 & 57,8 \\
\hline 2 & 66,0 & 34,0 \\
\hline 3 & 79,2 & 20,8 \\
\hline 4 & 75,0 & 25,0 \\
\hline \multicolumn{3}{|l|}{ Arroz } \\
\hline Todos os dias/semana & 73,8 & 26,2 \\
\hline Até 4 vezes/semana & 74,7 & 25,3 \\
\hline Nunca ou quase nunca & 34,0 & 66,0 \\
\hline \multicolumn{3}{|l|}{ Feijão } \\
\hline Todos os dias/semana & 74,5 & 25,5 \\
\hline Até 4 vezes/semana & 64,1 & 35,9 \\
\hline Nunca ou quase nunca & 64,0 & 36,0 \\
\hline \multicolumn{3}{|c|}{ Consumo semanal de verduras } \\
\hline Sim & 79,6 & 20,4 \\
\hline Não & 48,4 & 51,6 \\
\hline \multicolumn{3}{|l|}{ Consumo semanal de frutas } \\
\hline $\operatorname{Sim}$ & 89,8 & 10,2 \\
\hline Não & 54,3 & 45,7 \\
\hline
\end{tabular}

Doenças Crônicas por Inquérito Telefônico (VIGITEL), as autoras encontraram resultados que apontaram uma associação do tipo de alimentação com valores de IMC, sugerindo que as mulheres buscam a alimentação equilibrada ${ }^{12}$.

No presente estudo, a alimentação saudável e o consumo de verduras e frutas foi sempre mais prevalente entre aquelas que estavam em faixas etárias mais elevadas, principalmente acima dos 50 anos, e entre as que apresentaram maiores renda e escolaridade, resultados semelhantes a outros estudos ${ }^{34-36}$.

Uma possível limitação deste estudo, típica dos estudos transversais, seria o viés de causalidade reversa na associação entre frequência de consumo de frutas, legumes e verduras e estado nutricional, uma vez que as pessoas, principalmente com excesso de peso e obesidade, podem modificar seu consumo em função do estado nutricional.

Os resultados do presente estudo revelaram o excesso de peso como importante distúrbio nutricional entre as mulheres pobres de Campos 
Elíseos, demonstrando que mesmo em uma população com renda baixa, um maior nível de escolaridade tem impacto na prevenção deste agravo e nas escolhas alimentares. Estes achados apontam para a necessidade de maiores investimentos em estratégias de intervenção no campo das políticas públicas e programas voltados para os estratos menos favorecidos da população feminina.

\section{Colaboradores}

\section{Referências}

APM Lins participou da análise, interpretação, elaboração do artigo e discussão dos resultados. $\mathrm{R}$ Sichieri participou da fase de planejamento, metodologia e discussão dos resultados. WF Coutinho participou da fase de interpretação e discussão dos resultados. GE Ramos participou da análise, interpretação, elaboração do artigo e discussão dos resultados. MVM Peixoto participou da fase de interpretação e discussão dos resultados. VM Fonseca participou da análise, interpretação, elaboração do artigo e discussão dos resultados. Todos participaram da elaboração do texto.

1. Batista Filho M, Souza AI, Miglioli TC, Santos MC. Anemia e obesidade: um paradoxo da transição nutricional brasileira. Cad Saude Publica 2008; 24(2):247-257.

2. Instituto Brasileiro de Geografia e Estatística (IBGE). Pesquisa de orçamentos familiares 2008-2009: análise da disponibilidade domiciliar de alimentos e do estado nutricional no Brasil. Rio de Janeiro: IBGE; 2010. [site da internet]. [acessado 2012 nov 29]. Disponível em: http://www.ibge.gov.br/home/presidencia/ noticias/noticia_visualiza.php?id_noticia $=1648$ \&id_pagina $=1$.

3. Gigante DP, Dias-da-Costa JS, Olinto MTA, Menezes AMB, Macedo S. Obesidade da população adulta de Pelotas, Rio Grande do Sul, Brasil, e associação com nível sócio-econômico. Cad Saude Publica 2006; 22(9):1873-1879.

4. Alves V, Magalhães R. Obesidade e Pobreza: O Aparente Paradoxo - Um estudo com mulheres da Favela da Rocinha. Cad Saude Publica 2005; 21(6): 1792-1800.

5. World Health Organization (WHO). Preventing chronic diseases: a vital investment: WHO global report. Geneva: WHO; 2010.

6. Mendonça CP. Práticas alimentares e de atividade física de mulheres obesas atendidas em unidades de saúde pública do Município de Niterói: trajetórias e narrativas [tese]. Rio de Janeiro: Fiocruz, Escola Nacional de Saúde Pública; 2005.

7. Organización Mundial de la Salud, Organización de las Naciones Unidas para la Agricultura y la Alimentación. Un marco para la promoción de frutas $y$ verduras a nivel nacional. [site da internet]. [acessado 2012 nov 29]. Disponível em: http://www.who. int/dietphysicalactivity/reportSP\%20final.pdf.

8. Lock K, Pomerleau J, Causer L, Altmann DR, McKee M. The global burden of disease attributable to low consumption of fruit and vegetables: implications for the global strategy on diet. Bull World Health Organ 2005; 83(2):100-108.

9. Claro R M, Carmo HCE, Machado FMS, Monteiro CA. Renda, preço dos alimentos e participação de frutas e hortaliças na dieta. Rev Saude Publica 2007; 41(4):557-564.

10. World Health Organization (WHO). Diet, nutrition and the prevention of chronic diseases. Genève: WHO Technical Report Series; 2003. 
11. Liu RH. Health benefits of fruit and vegetables are from additive and synergistic combinations of phy tochemicals 1-4. Am J Clin Nutr 2003; 78(Supl. 3):517-520.

12. Sichieri R, Moura EC. Análise multinível das variações no índice de massa corporal entre adultos, Brasil, 2006. Rev Saude Publica 2009; 43(2):90-97.

13. Brasil. Ministério da Saúde (MS). Análise da Estratégia Global para alimentação Saudável, atividade física e saúde. Documento realizado pelo Grupo téc nico assessor instituído pela Portaria do Ministério da Saúde no 596, de 8 de abril de 2004.

14. Brasil. Ministério da Saúde (MS). Secretaria de Atenção à Saúde Coordenação-Geral da Política de Alimentação e Nutrição. Guia Alimentar para População Brasileira. Brasília: MS; 2005. (Série A. Normas e Manuais Técnicos).

15. Gordon CC, Chumlea WC, Roche AF. Stature, recumbert length, and weight. In: Lohman TG, Roche AF, Martorell R, editors. Anthropometric standardization reference manual. Champaign: Human kinetics Books; 1988. p. 3-8.

16. World Health Organization (WHO). Energy and Protein Requirements. Food and Agricultural Organization. United Nations University, 1985. Geneva: WHO; 1985.

17. Brasil. Ministério do Trabalho (MT). Lei n. 6.514 de 22 de dezembro de 1977. Altera o capítulo V do título II da Consolidação das Leis do Trabalho, relativas à segurança e Medicina do Trabalho, e dá outras providências. São Paulo: Lex Editor; 1977.

18. Ainsworth BE, Haskell WL, Whitt ME, Irwin ML, Swartz AM, Strath SJ, O'Brien WL, Bassett DR Jr, Schmitz KH, Emplaincourt PO, Jacobs DR Jr, Leon AS. Compendium of Physical Activities: an update of activity codes and MET intensities. Med Sci Sports Exerc 2000; 32(Supl. 9):S498-S504.

19. Bickel G, Nord M, Price C, Hamilton W, Cook J. Guide to measuring household fod security. Alexandria. Department of Agriculture Food and $\mathrm{Nu}-$ trition Service; 2000.

20. Victora CG, Huttly SR, Fuchs SC, Olinto MTA. The role of conceptual frameworks in epidemiological analysis: A hierarchical approach. Int J Epidemio 1997; 26(1):224-227.

21. Martuzzi M, Elliott P. Estimating the incidence rate ratio in cross-sectional studies using a simple alternative to logistic regression. Ann Epidemiol. 1998; 8(1):52-55.

22. Traissac P, Martin-Prével Y, Delpeuch B, Maire B. Régression logistique vs autres modèles linéaires généralizés pour l' estimation de rapports de prévalences. Rev Epidém. et Santé Publ 1999; 47:593-604.

23. Brasil. Ministério da Saúde (MS). Pesquisa Nacional de Demografia e Saúde da Criança e da Mulher. Brasília: MS; 2008.
24. Teichmann L, Olinto MTA, Costa JSD, Ziegler D. Fatores de risco associados ao sobrepeso e a obesidade em mulheres de São Leopoldo, RS. Rev Bras Epidemiol 2006; 9(3):360-973.

25. Bendixen H, Holst C, Sørensen TIA, Raben A, Bartels EM, Astrup A. Major increase in prevalence of overweight and obesity between 1987 and 2001 among danish adults. Obes Res 2004; 12(9):1464-1472.

26. Torrance GM, Hooper MD, Reeder BA. Trends in overweight and obesity among adults in Canada (1970-1992): evidence from national surveys using measured height and weight. Int J Obes Relat Metab Disord 2002; 26(6):797-804.

27. Caballero B. The global epidemic of obesity: an overview. Epidemiol Rev 2007; 29:1-5.

28. Popkin BM, Gordon-Larsen P. The nutrition transition: worldwide obesity dynamics and their determinants. Int J of Obesity 2004; 28(Supl.):S2-S9.

29. Ogden CL, Yanovski SZ, Carroll MD, Flegal KM. The epidemiology of obesity. Gastroenterology 2007; 132(6):2087-2102.

30. Instituto Brasileiro de Geografia e Estatística (IBGE). Pesquisa Nacional por Amostra Domiciliar. Rio de Janeiro: IBGE; 2007.

31. Monteiro CA, Conde WL, Castro, IRR. A tendência cambiante da relação entre escolaridade e risco de obesidade no Brasil (1975-1997). Cad Saude Publica 2003; 19(Supl.):S67-S75.

32. Monteiro CA, Moura EC, Conde WL, Popkin BM. Socioeconomic status and obesity in adult populations of developing countries: a review. Bull World Health Organ 2004; 82(12):940-946.

33. Neutzling MB, Rombaldi AJ, Azevedo MR, Hallal PC. Fatores associados ao consumo de frutas, legumes e verduras em adultos de uma cidade no Sul do Brasil. Cad Saude Publica 2009; 25(11):2365-2374.

34. Figueiredo IC, Jaime PC, Monteiro CA. Factors associated with fruit and vegetable intake among adults of the city of Sao Paulo, Southeastern Brazil. Rev Saude Publica 2008; 42(5):777-785.

35. Brasil. Ministério da Saúde (MS). Vigilância de fatores de risco e proteção para doenças crônicas por inquérito telefônico - VIGITEL. Brasília: MS; 2009.

36. Jaime PC, Monteiro CA. Fruit and vegetable intake by Brazilian adults, 2003. Cad Saude Publica 2005; 21(Supl.):S19-S24.

Artigo apresentado em 09/10/2011

Aprovado em 10/11/201

Versão final aprovada em 21/01/2012 